\title{
Spectrum Estimation of Interleaved Pulse Trains
}

\author{
Robert J. Orsi, Student Member, IEEE, John B. Moore, Fellow, IEEE, and Robert E. Mahony, Member, IEEE
}

\begin{abstract}
In this paper, we consider signals consisting of a finite though unknown number of periodic time-interleaved pulse trains. For such signals, we present a novel approach for determining both the number of pulse trains present and the frequency of each pulse train. Our approach requires only the time of arrival data of each pulse. It is robust to noisy time of arrival data and missing pulses and, above all, is very computationally efficient. If $N$ is the number of pulses being processed, the computation required is of the order of $N \log N$.
\end{abstract}

Index Terms-Pulse train analysis.

\section{INTRODUCTION}

A PERIODIC pulse train consists of a sequence of periodically spaced pulses. A single channel receiver will often receive periodic pulse trains from a number of sources simultaneously. The superposition of all the received pulse trains is known as an interleaved pulse train. The process of determining the number of pulse trains present in this signal and associating each received pulse with a source is termed pulse train deinterleaving. This process relies on the assumption that the different pulse train sources have different characteristics such as period of pulse emission. One application of pulse train deinterleaving is in radar detection [5]. Potential applications include computer communications and neural systems.

Typical approaches to pulse train deinterleaving are sequential search [1] and histogramming [1], [2]. A practical disadvantage of these algorithms is the computational effort they require. If $N$ is the number of pulses being processed, computations are of the order of $N^{2}$ [4].

A recent novel approach to pulse train deinterleaving is given in [3], where the problem is first formulated as a stochastic discrete-time dynamic linear model. Like sequential search and histogramming, this method is quite computationally expensive.

Rather than trying to deinterleave a received interleaved pulse train directly, we focus solely on determining the number of pulse trains present and the frequency of each pulse train. We term this information the interleaved pulse train spectrum. We present a novel (nonlinear) approach for estimating the

Manuscript received October 22, 1996; revised September 8, 1998. The associate editor coordinating the review of this paper and approving it for publication was Prof. Peter C. Doerschuk.

R. J. Orsi is with the Department of Electrical and Electronics Engineering, The University of Melbourne, Parkville, Australia.

J. B. Moore is with the Department of Systems Engineering, RSISE, Australian National University, Canberra, Australia.

R. E. Mahony is with the Centre de Recherche, Université de Technologie de Compiègne, Compiègne, France.

Publisher Item Identifier S 1053-587X(99)03691-0. spectrum of a signal consisting of a finite though an unknown number of periodic time-interleaved pulse trains. Only the time of arrival data of each pulse is assumed to carry information, and only this data is used in our algorithm. No knowledge of any other pulse train characteristics such as pulse energy is required, nor is prior knowledge of the transmitter characteristics required. A great advantage of our scheme is that if $N$ is the number of pulses being processed, computations are of the order $N \log N$. Note that once the interleaved pulse train spectrum is known, it is a relatively easy task to deinterleave the received signal using standard methods such as those used after histogramming [1].

This paper is structured as follows. A problem formulation is first presented, followed by an overview of the proposed scheme. The remainder of the paper then discusses aspects of the approach in greater depth. First, an analysis of a special class of nongeneric pulse train sequences that satisfy various simplifying assumptions is undertaken. Using insight gained from this nongeneric case, some simulation results for the generic case are presented and discussed. These results include considering how the length of the data set, pulse time of arrival noise, missing pulses, and staggered pulse trains effect the accuracy of the scheme. The paper ends with some concluding remarks.

\section{PROBlem Formulation AND APPROACH}

Consider $M$ periodic pulse train sources. Let $T_{i}, f_{i}$, and $\phi_{i}$ denote, respectively, the period, frequency, and phase of the $i$ th source. The received interleaved signal consists of the superposition of the $M$ pulse trains produced by these sources. Let $t_{0}, t_{1}, \cdots, t_{N}$ denote the times of arrival of $N+1$ consecutive pulses in this signal nominally setting $t_{0}:=0$. The problem is as follows:

Problem: Given $t_{0}, \cdots, t_{N}$, determine both the number of pulse trains present and the frequency of each pulse train.

The first step in the proposed scheme is to calculate

$$
x(n):=e^{j\left(2 \pi / t_{N}\right) t_{n n}} \quad \text { for } n=0, \cdots, N-1
$$

where $j:=\sqrt{-1}$. The signal $x(n)$ can be thought of as taking the interval $\left[t_{0}, t_{N-1}\right]$, containing the first $N$ pulse times of arrival, normalizing its length to approximately $2 \pi$, and then wrapping this normalized interval around the unit circle. Note that as mentioned before, $t_{0}=0$.

The next step is to take the $N$-length discrete Fourier transform (DFT) of (2.1). The magnitude of this transformed signal contains the information necessary to determine the interleaved pulse train spectrum. That is, it contains the 
information necessary to 1) determine how many pulse trains are present and 2) make a good estimate of their frequencies. The phase response seems to contain little information. Redundant information within the magnitude signal can be used to improve confidence of results.

By choosing appropriate data lengths, the proposed scheme can employ the fast Fourier transform (FFT). Hence, the computational cost of the scheme is of the order of $N \log N$.

Last, note that the proposed scheme is nonlinear.

\section{A Nongeneric SPeCial CASE}

The nonlinear nature of the proposed scheme makes its mathematical analysis difficult. In this section, we consider a class of nongeneric pulse trains that satisfy various simplifying assumptions and make a mathematical analysis of the scheme possible. As discussed in the next section, this analysis provides valuable insight into the generic case.

Let $\mathbb{Q}$ denote the rational numbers. It is assumed that the pulse trains considered in this section satisfy the following properties.

P1) The period of each pulse train is rational, that is, $T_{i} \in \mathbb{Q}, i=1, \cdots, M$.

P2) The phase of each pulse train is zero, that is, $\phi_{i}=$ $0, i=1, \cdots, M$.

In real-world situations, some pulses will not be detected due to pulses from different sources arriving at the receiver at exactly or nearly exactly the same time. For the present purposes of analysis, we make an idealization in the follwing assumption.

Assumption 3.1: All emitted pulses are detected by the receiver, and there are no missing pulses.

Simulations later in the paper will show that the algorithm is robust to small percentages of missing pulses and, hence, that Assumption 3.1 is not crucial to the operation of the algorithm.

Properties P1) and P2) and Assumption 3.1 imply that if the received signal contains a sufficiently large number of pulses, it will be periodic. It is assumed that a sufficiently large number of pulses have been received such that this is the case. The period of the received signal will be denoted by $T$.

In addition to properties P1) and P2), we have the following property.

P3) The received signal consists of exactly an integer number of overall signal periods $T$.

Let $r_{i}$ denote the number of pulses from pulse train $i$ appearing in one period of the received signal. Then

$$
T=T_{1} r_{1}=\cdots=T_{M} r_{M}
$$

and the total number of pulses in one period of the received signal is

$$
N_{T}:=\sum_{i=1}^{M} r_{i} .
$$

Remark 3.2: It is assumed that the pulse time of arrival data $t_{0}, \cdots, t_{N}$ is noise free.

Let $\mathbb{Z}$ denote the integers. The prior assumptions imply that $N / N_{T} \in \mathbb{Z}$ and that

$$
t_{N}=\frac{N}{N_{T}} T .
$$

Theorem 3.3: Consider a signal consisting of $M$ interleaved pulse trains satisfying properties $\mathrm{P} 1), \mathrm{P} 2)$, and P3). Let $x(n)$ be defined as in (2.1), and let $X(k), k=0, \cdots, N-1$ denote its discrete Fourier transform. Then, defining $k^{\prime}=k-1$

$$
X\left(k^{\prime}\right)=\left\{\begin{array}{l}
\frac{N}{N_{T}} \sum_{l=0}^{N_{T}-1} e^{j(2 \pi / N)\left(\left(N_{T} / T\right) t_{l}-k^{\prime} l\right)} \\
\quad \text { if } \quad k^{\prime}=\frac{p N}{N_{T}}, p=0, \cdots, N_{T}-1 \\
0, \quad \text { otherwise. }
\end{array}\right.
$$

Furthermore, for $p=r_{j},\left(k^{\prime} / t_{N}\right)$ equals $f_{j}$, which is the frequency of the $j$ th pulse train.

Proof: By definition

$$
\begin{aligned}
X(k) & =\sum_{n=0}^{N-1} x(n) e^{-j k(2 \pi / N) n} \\
& =\sum_{n=0}^{N-1} e^{j\left(2 \pi / t_{N}\right) t_{n}} e^{-j k(2 \pi / N) n} .
\end{aligned}
$$

Replacing $n$ by $m N_{T}+l$ and noting that P2) implies that $t_{n}=t_{m N_{T}+l}=m T+t_{l}$

$$
\begin{aligned}
X(k)= & \sum_{m=0}^{\left(N / N_{T}\right)-1} \sum_{l=0}^{N_{T}-1} e^{j\left(2 \pi / t_{N}\right)\left(m T+t_{l}\right)} \\
\cdot & e^{-j k(2 \pi / N)\left(m N_{T}+l\right)} \\
= & \sum_{m=0}^{\left(N / N_{T}\right)-1} \sum_{l=0}^{N_{T}-1} e^{j 2 \pi\left(N_{T} / N T\right)\left(m T+t_{l}\right)} \\
= & \sum_{m=0}^{\left(N / N_{T}\right)-1} \sum_{l=0}^{-j k(2 \pi / N)\left(m N_{T}+l\right)} e^{j(2 \pi / N)\left((1-k) N_{T} m+\left(N_{T} / T\right) t_{l}-k l\right)} \\
= & \sum_{l=0}^{N_{T}-1}\left[\sum_{m=0}^{\left(N / N_{T}\right)-1}\left(e^{\left.j(2 \pi / N)(1-k) N_{T}\right)^{m}}\right]\right. \\
& \cdot e^{j(2 \pi / N)\left(\left(N_{T} / T\right) t_{l}-k l\right)} \cdot
\end{aligned}
$$


Consider the summation in square brackets in the line above. Letting

$$
\begin{aligned}
\sum_{m=0}^{\left(N / N_{T}\right)-1}\left(e^{j(2 \pi / N)(1-k) N_{T}}\right)^{m} & =\sum_{m=0}^{j(2 \pi / N)(1-k) N_{T}} \\
& = \begin{cases}\frac{z^{N / N_{T}}-1}{N^{z-1}}, & \text { if } z \neq 1 \\
\frac{N_{T}}{N_{T}}, & \text { if } z=1 .\end{cases}
\end{aligned}
$$

Note that $z^{N / N_{T}}=1$ and, hence, that

$$
\sum_{m=0}^{\left(N / N_{T}\right)-1}\left(e^{j(2 \pi / N)(1-k) N_{T}}\right)^{m}= \begin{cases}0, & \text { if } z \neq 1 \\ \frac{N}{N_{T}}, & \text { if } z=1 .\end{cases}
$$

In addition, note that

$$
\begin{aligned}
& z=1 \Leftrightarrow \frac{1-k}{N} N_{T}=-p \\
& \text { where } p \in \mathbb{Z} \quad \text { and } k \in\{0, \cdots, N-1\} \\
& \Leftrightarrow k-1=\frac{p N}{N_{T}}, \quad p=0, \cdots, N_{T}-1 .
\end{aligned}
$$

Note that $k$ above is indeed always an integer as $N / N_{T} \in \mathbb{Z}$.

Replacing $k-1$ with $k^{\prime}$, the DFT of $x(n)$ can now be seen to be the expression given in the theorem statement. Furthermore, by $(3.2)$

$$
\frac{k^{\prime}}{t_{N}}=\frac{p N / N_{T}}{N T / N_{T}}=\frac{p}{T}
$$

and for $p=r_{j}$, (3.1) implies that

$$
\frac{k^{\prime}}{t_{N}}=\frac{r_{j}}{T_{j} r_{j}}=\frac{1}{T_{j}}=f_{j}
$$

Theorem 3.3 shows that the $N$-length DFT of $x(n)$ is nonzero at, at most, $N_{T}$ points. Furthermore, $M$ of these possibly nonzero points correspond to the $M$ pulse train frequencies. Additionally, if $f$ is a pulse train frequency, the theorem predicts the existence of harmonics at $2 f, 3 f, \cdots$.

\section{A. The Nongeneric Case: A Simulation Example}

The proposed methodology was applied to a signal satisfying properties P1)-P3). The signal consisted of $M=3$ interleaved pulse trains with respective frequencies $f_{1}=0.25$ $\mathrm{Hz}, f_{2}=0.75 \mathrm{~Hz}$, and $f_{3}=0.8 \mathrm{~Hz}$. The magnitude of the signal produced by the DFT is shown in Fig. 1.

As predicted, the magnitude plot contains only a small number of nonzero values uniformly spaced in frequency. How the interleaved pulse train spectrum is identified from such a magnitude plot will be discussed in the next section, which

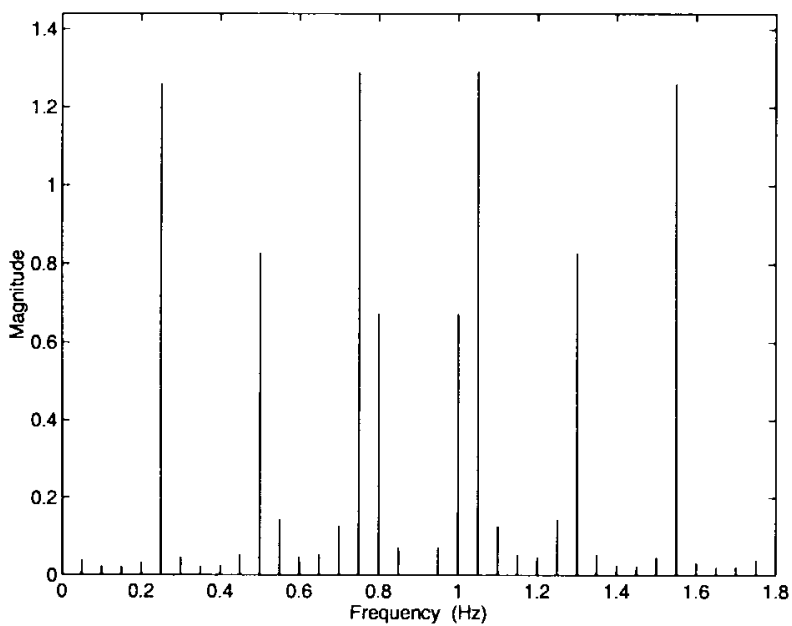

Fig. 1. Nongeneric magnitude plot.

deals with the generic case. For the moment, notice that the $M$ largest values produced by the proposed scheme do not necessarily correspond to the $M$ pulse trains present. Despite this, notice that, for this example at least, $M=3$ of the larger magnitudes present do correspond to pulse train frequencies.

Although it is not apparent from Fig. 1, the DFT magnitude at $0 \mathrm{~Hz}$ is very large and is approximately equal to $N$. This term is an artifact of the processing method and is ignored.

\section{The Generic CASE}

In this section, a generic case simulation is presented and discussed. The time-of-arrival data used in this simulation is not corrupted by noise, and the data does not contain any missing pulses. (The effects of noisy time of arrival data and missing pulses are discussed in Section V.)

The simulated signal consists of ten interleaved pulse trains. The frequencies of the pulse trains were chosen arbitrarily and are listed in Table I. Each pulse train has a random phase, and the number of pulses used in the simulation is $N=2^{12}=4096$. The magnitude plot produced by applying our approach is shown in Fig. 2. Fig. 3 highlights the output in the frequency range $1-6 \mathrm{kHz}$. Here, it can be seen that the ten largest magnitudes in the spectrum correspond to the ten pulse trains. (As in the nongeneric case, the spectrum contains a large term at $0 \mathrm{~Hz}$, which is ignored.)

If the original signal consists of $M$ interleaved pulse trains, the number of pulses processed $N$ will approximately be equal to $t_{N}\left(f_{1}+\cdots+f_{M}\right)$. The number of pulse trains present is determined by assuming that the $m$ largest magnitudes correspond to pulse trains. Starting with $m=1, m$ is incremented until $t_{N}\left(\hat{f}_{1}+\cdots+\hat{f}_{m}\right)$ is approximately equal to $N$. (The frequencies $\hat{f}_{1}, \cdots, \hat{f}_{m}$ are estimates of $f_{1}, \cdots, f_{m}$.) If no such $m$ can be found, it means the $M$ largest magnitudes in the spectrum do not correspond to the $M$ pulse trains present.

For our example, actual versus estimated pulse train frequencies are presented in Table I.

Before proceeding, we note that the behavior of the algorithm as described above is typical if two or more pulse trains are present. On the other hand, in the case that only a single 
TABLE I

Actual Versus Estimated Pulse Train Frequencies

\begin{tabular}{ccc}
\hline PT No. & Actual Freq. (kHz) & Estimated Freq. (kHz) \\
\hline 1 & 1.2980 & 1.2981 \\
2 & 1.7400 & 1.7409 \\
3 & 2.0658 & 2.0635 \\
4 & 2.0944 & 2.0935 \\
5 & 2.7183 & 2.7164 \\
6 & 3.0000 & 3.0015 \\
7 & 3.3416 & 3.3392 \\
8 & 4.1416 & 4.1421 \\
9 & 4.8200 & 4.8174 \\
10 & 5.5100 & 5.5077 \\
\hline
\end{tabular}

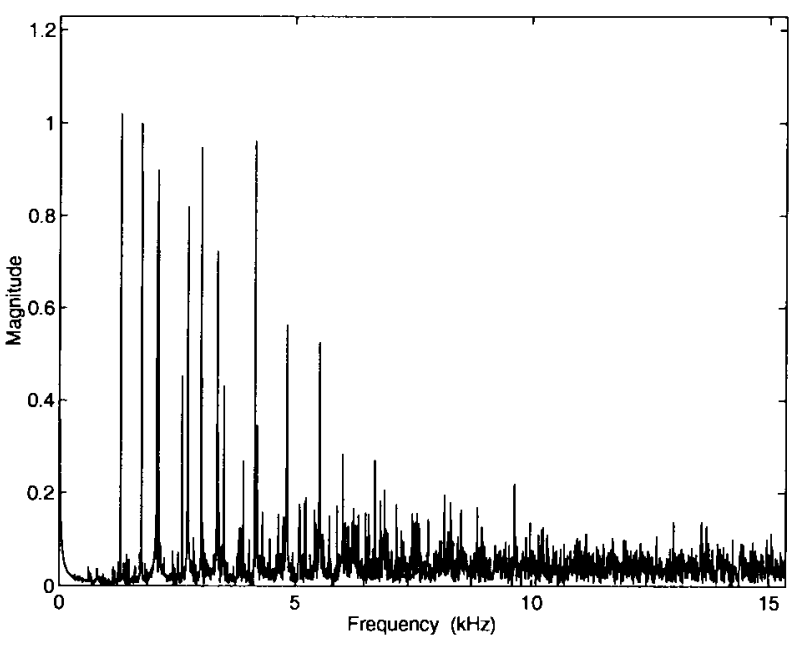

Fig. 2. Generic magnitude plot $(N=4096)$.

pulse train is present, the algorithm behaves slightly differently in that it produces a magnitude plot containing only a large magnitude at $0 \mathrm{~Hz}$ (which in normal processing is ignored). In such a situation (an estimate of), the frequency of the pulse train will be given by the highest frequency bin in the FFT.

\section{A. Additional Processing}

As Fig. 3 demonstrates, the proposed scheme can produce a magnitude plot that contains magnitudes of a substantial size that do not correspond to pulse trains. Instead of trying to determine the interleaved pulse train spectrum from such a magnitude plot, the magnitude signal can be further processed in such a way that many of the magnitudes that do not correspond to pulse trains can be removed. This processing removes many of the artifacts present and leads to a more reliable estimate of the interleaved pulse train spectrum. We now discuss how this additional processing is done.

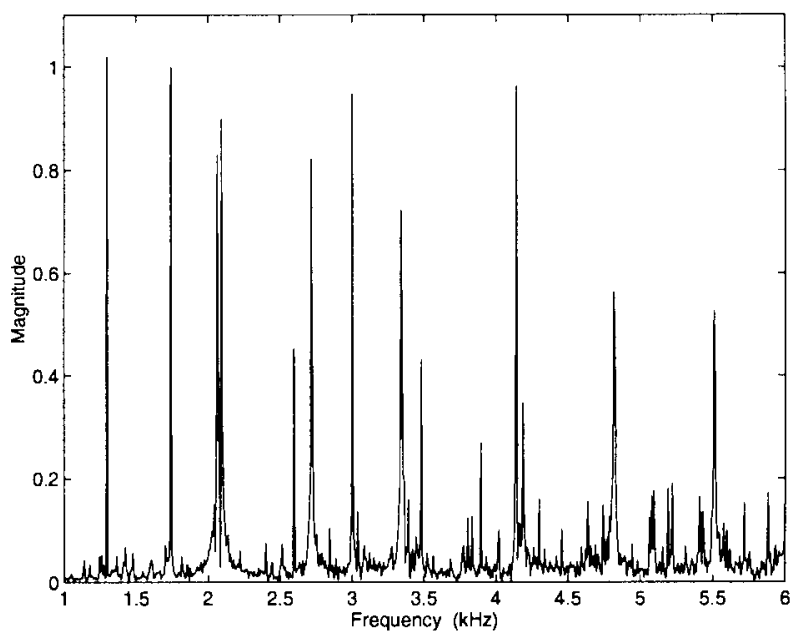

Fig. 3. Region of interest from Fig. 2.

As mentioned previously, at least for the nongeneric case, if $f$ is a pulse train frequency, Theorem 3.3 predicts the existence of harmonics at $2 f, 3 f, \cdots$. Simulations indicate that such harmonics also appear in the generic case and that, in fact, they make up the majority of spurious pulse train magnitudes. Simulations also indicate that the largest value in a magnitude plot always corresponds to a pulse train. ${ }^{1}$ Additional processing starts by assuming the largest magnitude present corresponds to a pulse train. The estimated frequency of this pulse train $\hat{f}_{1}$ is taken to be the frequency corresponding to this magnitude. Any magnitudes at $2 \hat{f}_{1}, 3 \hat{f}_{1}, \cdots$ are assumed to be harmonics of this pulse train and are removed from the magnitude plot. (In practice, for reasons of robustness, one or two frequency bins directly either side of the DFT bin corresponding to each harmonic are also removed.) The sum of the magnitudes of these harmonics are then added to the magnitude at frequency $\hat{f}_{1}$. This process is then repeated on the second largest magnitude present, and then on the next largest magnitude, and so on, until $t_{N}\left(\hat{f}_{1}+\cdots+\hat{f}_{m}\right)$ approximately equals $N$. (In practice, when deciding which is the next largest magnitude, not only are the bins corresponding to previously identified pulse trains ignored, but the two bins either side of such bins are also ignored. This helps to ensure that if a pulse train magnitude is spread over more than one bin, the pulse train is not incorrectly identified as a multiple pulse train.) The result of such additional processing for our example is shown in Fig. 4.

It is important to note that the additional processing discussed in this subsection incurs negligible additional computational cost.

\section{B. When All Else Fails ...}

If the proposed scheme (with additional processing) fails to properly identify the interleaved pulse train spectrum, for example, if no $m$ can be found such that $t_{N}\left(\hat{f}_{1}+\cdots+\hat{f}_{m}\right)$ is

\footnotetext{
${ }^{1}$ A magnitude plot may contain two frequencies of equal largest magnitude; see, for example, Fig. 1. In this case, at least one of these largest magnitudes has always been observed to correspond to a pulse train. Equal largest magnitudes have only been observed in nongeneric case simulations.
} 


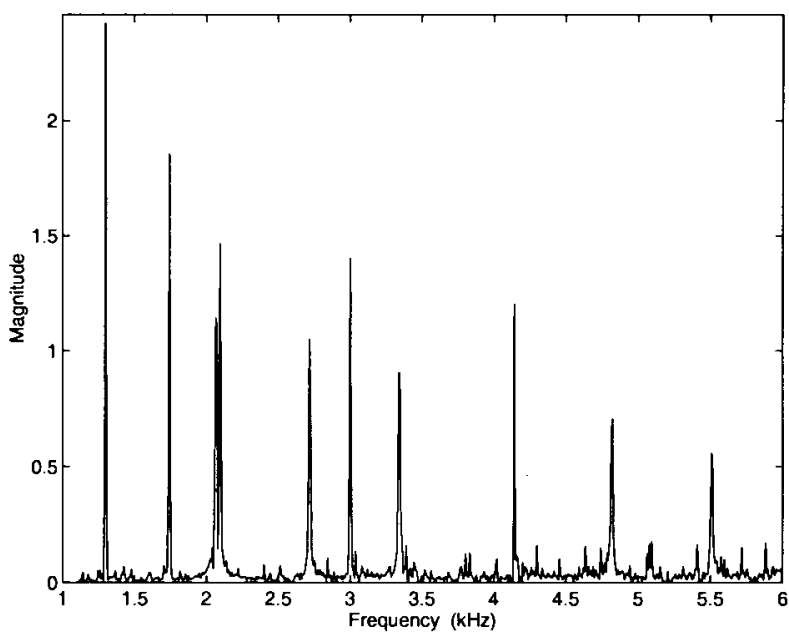

Fig. 4. Magnitude plot after additional processing.

approximately equal to $N$, the spectrum can be identified in the following manner. As previously mentioned, simulations indicate that the largest magnitude in a magnitude plot always corresponds to a pulse train. (As usual, the large magnitude at 0 $\mathrm{Hz}$ is ignored.) Having identified the frequency corresponding to the largest magnitude, standard methods [1] can be used to deinterleave the corresponding pulse train. By deinterleave, we mean that all pulses in the received interleaved signal that are members of the identified pulse train can be removed. This produces a new interleaved signal with one fewer present pulse train than the original. The proposed scheme can then be applied to this new signal, and another pulse train can be identified and deinterleaved. This process can be repeated until all pulse trains are identified.

The method described in this subsection involves considerably more computational effort than the approach described earlier. As a consequence, it should only be used as a last resort. No results presented in this paper are based on such processing.

\section{FURTHER ANALYSIS}

In this section, we continue to look at the generic case and consider how the length of the data set, pulse time of arrival noise, missing pulses, and staggered pulse trains effect results. All pulse train data used in this section is based on the interleaved pulse train signal used in Section IV.

\section{A. Decreasing $N$}

In this subsection, we consider the effect decreasing $N$, that is, of using a smaller number of pulses.

The simulation presented in Section IV used $N=2^{12}=$ 4096 pulses (see Fig. 3). The output produced by using a smaller number of pulses $N=2^{10}=1024$ is shown in Fig. 5 .

This figure is a plot of the results without additional processing. As would be expected, using a smaller number of pulses leads to a loss in resolution. Overall, the results are still very good, although pulse trains 3 and 4 , which are very

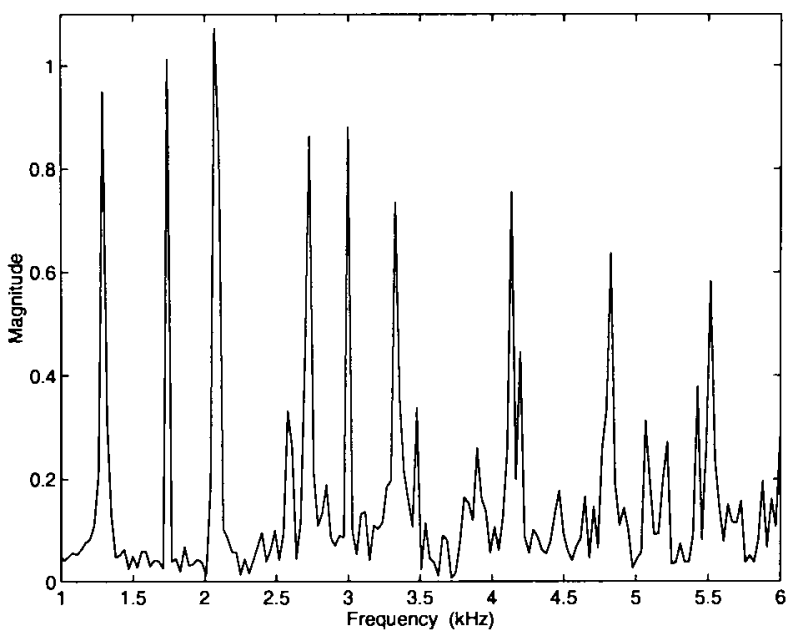

Fig. 5. Magnitude plot using a decreased number of pulses: $N=1024$.

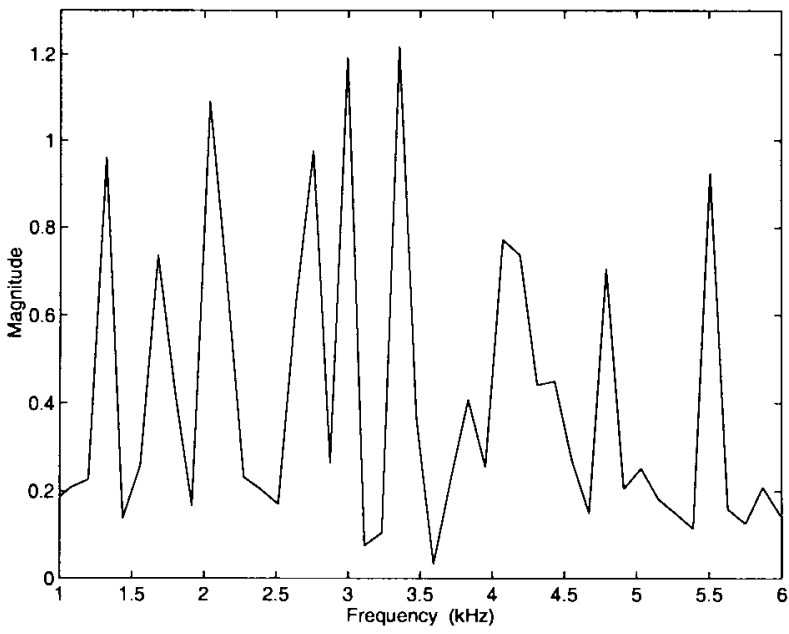

Fig. 6. Magnitude plot with $N=256$.

close in frequency ( 2.0658 and $2.0944 \mathrm{kHz}$, respectively), have not been distinguished and have been incorrectly identified as a single pulse train.

The effect of further reducing $N$ is demonstrated in Fig. 6, in which $N=2^{8}=256$. As can be seen, resolution has been greatly reduced, and in practice, a larger value of $N$ would be required if accurate estimates of the pulse train frequencies were required. Notice, however, that despite the poor resolution, nine prominent spikes are present, representing nine of the ten pulse trains present.

Overall, Figs. 3, 5, and 6 demonstrate that performance degrades gracefully as $N$ is decreased.

\section{B. Noisy Time of Arrival Data}

In this subsection, we consider the effect of noisy time-ofarrival data. Time-of-arrival noise is modeled as zero mean Gaussian noise and, as in Section IV, we use $N=4096$.

The output produced using our approach for a noise standard deviation of $0.025 \mathrm{~s}$ is shown in Fig. 7. Fig. 8 shows the results after additional processing. All ten pulse trains are correctly identified, and the estimated pulse train frequencies produced are given in Table II. Table II also lists actual pulse train 


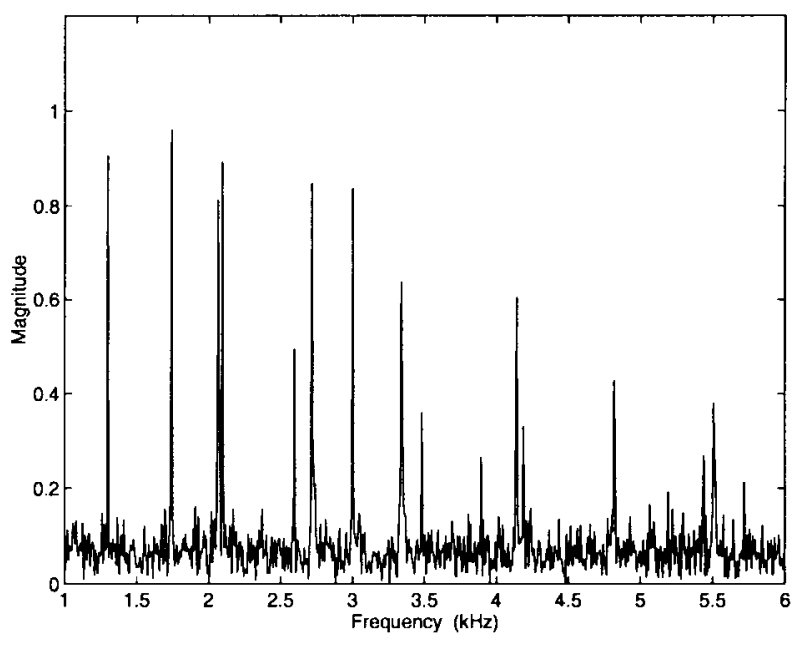

Fig. 7. Magnitude plot for data with noisy times of arrival. Noise std. dev. $=0.025 \mathrm{~s}$.

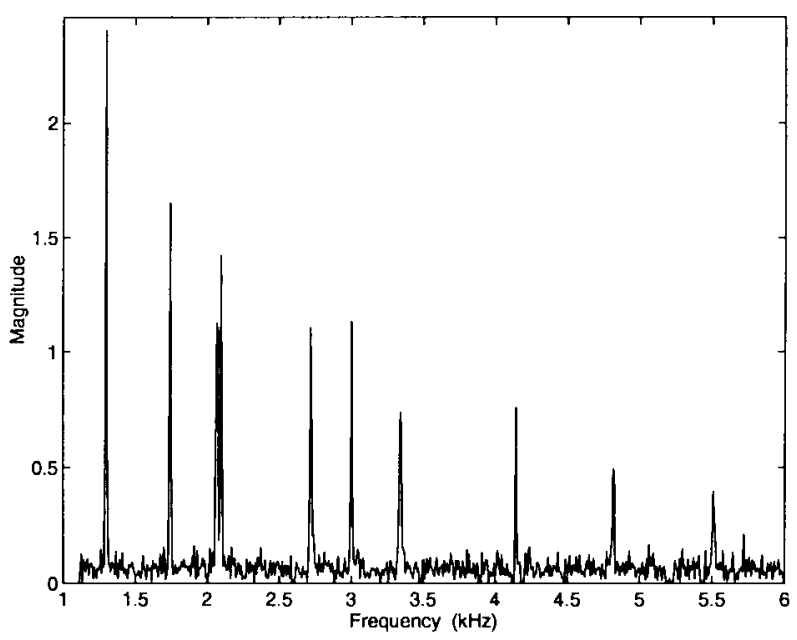

Fig. 8. Magnitude plot given in Fig. 7 after additional processing.

frequencies for convenience of comparison. Observe that for this level of noise, results are just as good as in the noise-free case given in Section IV.

Fig. 9 shows the output produced for a noise standard deviation of $0.05 \mathrm{~s}$. In this case, nine of the ten pulse train frequencies were correctly identified; however, four spurious frequencies were also identified. The frequency estimates in kilohertz were 1.2979, 1.4179, 1.5529, 1.7405, 2.0256, 2.0631, 2.0931, 2.7158, 2.8508, 3.0008, 3.3384, 4.1412, and 4.8238.

From the figures discussed above, it can be seen that increased time-of-arrival noise leads to greater noise in the pulse train magnitude plots. This increase in noise in turn leads to a decrease in performance. Note that although it is not shown here, when processing noisy data, results can be improved by increasing $N$.

\section{Missing Pulses}

In this subsection, we consider the effect of missing pulses. The signal processed was the same as the one used in Section IV, except that each pulse was given a probability of $1 \%$ of not been being present. As before, $N=4096$. Fig. 10
TABLE II

Actual Versus Estimated Pulse Train Frequencies: Time of Arrival Noise std. dev. $=0.025$

\begin{tabular}{ccc}
\hline PT No. & Actual Freq. (kHz) & Estimated Freq. (kHz) \\
\hline 1 & 1.2980 & 1.2983 \\
2 & 1.7400 & 1.7411 \\
3 & 2.0658 & 2.0638 \\
4 & 2.0944 & 2.0938 \\
5 & 2.7183 & 2.7167 \\
6 & 3.0000 & 3.0019 \\
7 & 3.3416 & 3.3396 \\
8 & 4.1416 & 4.1426 \\
9 & 4.8200 & 4.8180 \\
10 & 5.5100 & 5.5085 \\
\hline
\end{tabular}

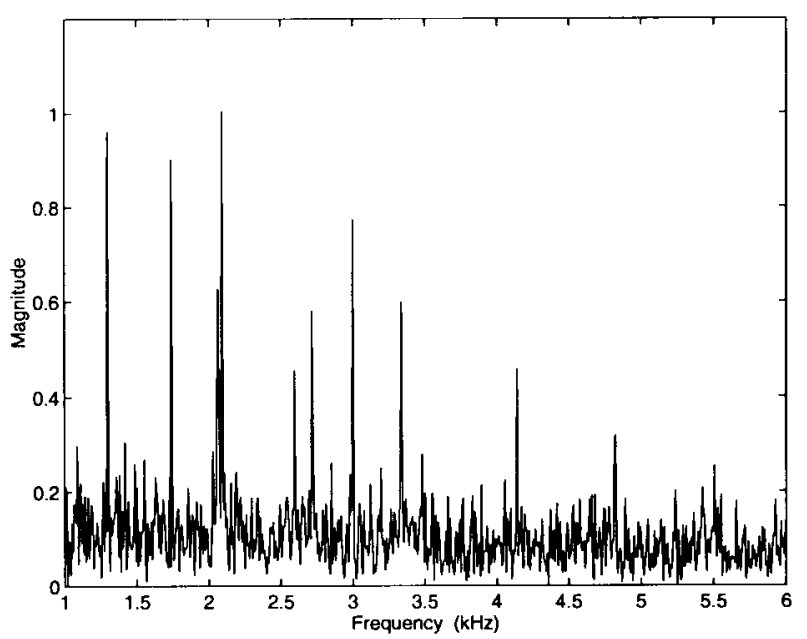

Fig. 9. Magnitude plot for data with noisy times of arrival. Noise std. dev. $=0.05 \mathrm{~s}$.

is a plot of the output produced after additional processing. Although pulse trains 3 and 4 cannot visually be distinguished from Fig. 10, our approach correctly identifies the ten pulse trains present, and the estimates of their frequencies are given in Table III.

\section{Staggered Pulse Trains}

In this subsection, we consider the effect of some emitters having staggered pulse repetition intervals. The signal processed was the same as the one used in Section IV, except that single copies of pulse trains 5, 6, and 10 were made, given a random offset that was added to the original signal.

The magnitude plot is shown in Fig. 11. As can be seen, the plot looks much the same as Fig. 3. Although this is quite encouraging and almost all simulations with staggered pulse trains look much the same, on occasion, the magnitude plot 


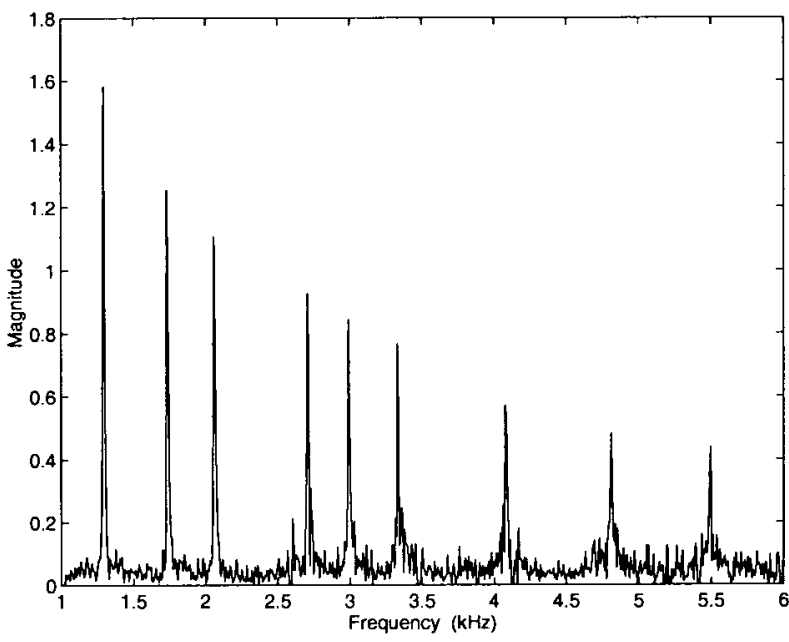

Fig. 10. Magnitude plot of data with $1 \%$ of pulses missing after additional processing.

TABLE III

Actual Versus Estimated Pulse Train FreQuencies For Data with $1 \%$ Missing PUlses

\begin{tabular}{ccc}
\hline PT No. & Actual Freq. (kHz) & Estimated Freq. (kHz) \\
\hline 1 & 1.2980 & 1.3018 \\
2 & 1.7400 & 1.7406 \\
3 & 2.0658 & 2.0679 \\
4 & 2.0944 & 2.0977 \\
5 & 2.7183 & 2.7225 \\
6 & 3.0000 & 3.0052 \\
7 & 3.3416 & 3.3474 \\
8 & 4.1416 & 4.1656 \\
9 & 4.8200 & 4.8277 \\
10 & 5.5100 & 5.5195 \\
\hline
\end{tabular}

produced has contained pulse trains with substantially reduced magnitudes. Why this is occurring is not clear.

\section{AdDitional COMMENTS AND CONCLUDing REMARKS}

The most important property of the proposed scheme is that it is computationally efficient. Computations are of the order of $N \log N$. Other typical deinterleaving methods, such as sequential search [1] and histogramming [1], [2], require order- $N^{2}$ computations [4].

The proposed methodology is also quite robust to noise. In Section V, it was shown that the performance of the proposed scheme degrades gracefully as pulse time-of-arrival noise is introduced and increased and that it is robust to missing pulses.

Simulations also indicate that magnitudes corresponding to lower frequency pulse trains tend to be larger than the magnitudes of pulse trains with comparatively higher frequencies. In

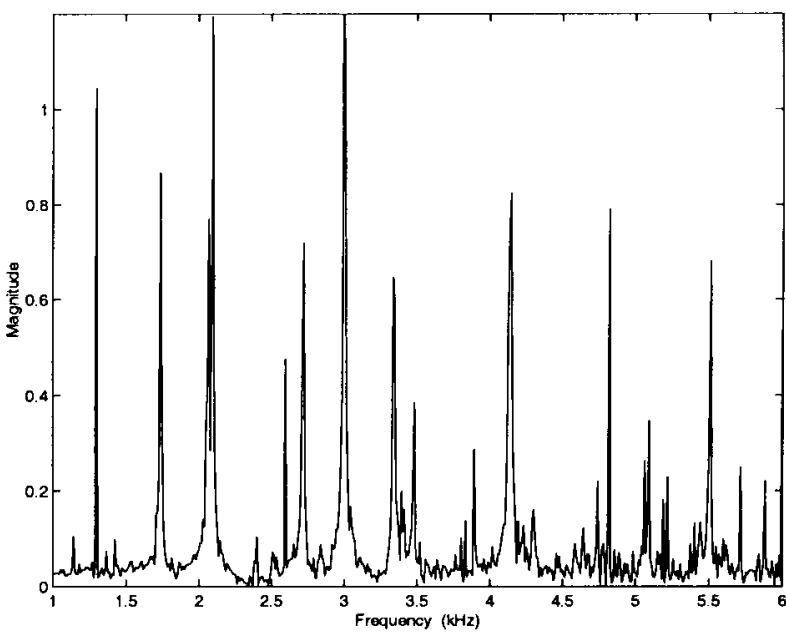

Fig. 11. Magnitude plot of data containing staggered pulse trains.

fact, if the ratio of largest to smallest pulse train frequencies present in an interleaved signal is too large, the spectrum magnitudes corresponding to the high-frequency pulse trains become submerged in noise. How large this ratio can be is dependent on $N$, and its size increases as $N$ is increased. If the proposed method is having trouble detecting high-frequency pulse trains, one option to try to improve detection would be to increase $N$. Note that increasing $N$ also increases the accuracy of frequency estimation.

Commonly used algorithms such as sequential search also suffer significant degradation of performance when the ratio of pulse train frequencies becomes too large. Since these existing methods identify high-frequency pulse trains most effectively, it is believed the proposed scheme could be used to compliment an existing algorithm for deinterleaving signals with pulse train frequency ratios exceeding these levels.

\section{REFERENCES}

[1] H. K. Mardia, "New techniques for the deinterleaving of repetitve sequences," Proc. Inst. Elect. F, vol. 136, pp. 149-154, 1989.

[2] D. J. Milojević and B. M. Popović, "Improved algorithm for the deinterleaving of radar pulses," Proc. Inst. Elect. F, vol. 139, pp. 98-104, 1992

[3] J. B. Moore, and V. Krishnamurthy, "Deinterleaving pulse trains using discrete-time stochastic dynamic-linear models," IEEE Trans. Signal Processing, vol. 42, pp. 3092-3102, Nov. 1994.

[4] J. Perkins and I. Coat, "Pulse train deinterleaving via the Hough transform," in Proc. Int. Conf. Acoust., Speech, Signal Process., vol. 3, 1994, pp. 197-200.

[5] R. G. Wiley, Electronic Intelligence: The Analysis of Radar Signals. Norwood, MA: Artech House.

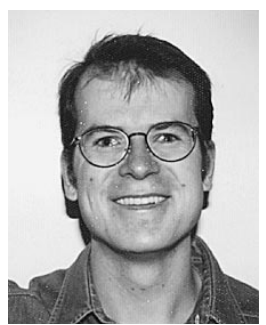

Robert J. Orsi (S'95) was born in Canberra, Australia, in April 1972. He received the B.S., B.E., and M.E. degrees, all from the Australian National University, Canberra, in 1992, 1994, and 1996 respectively. $\mathrm{He}$ is currently pursuing the $\mathrm{Ph} . \mathrm{D}$. degree in electrical engineering at the University of Melbourne, Melbourne, Australia.

His research interests are in nonlinear control. 


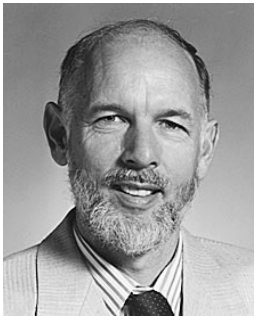

John B. Moore (F'79) was born in China in 1941. He received the B.S. and M.S. degrees in electrical engineering in 1963 and 1964, respectively, and the $\mathrm{Ph} . \mathrm{D}$. degree in electrical engineering from the University of Santa Clara, Santa Clara, CA, in 1967.

He was appointed Senior Lecturer at the Electrical Engineering Department, University of Newcastle, Callaghan, Australia, in 1967, promoted to Associate Professor in 1968, and Full Professor (personal chair) in 1973. He was Department Head from 1975 to 1979. In 1982, he was appointed Professional Fellow in the Department of Systems Engineering, Research School of Physical Sciences, Australian National University, Canberra, and promoted to Professor in 1990. He has been Head of the Department since 1992. The department is now located in the Research School of Information Sciences and Engineering. He has held visiting academic appointments at University of Santa Clara (1968); University of Maryland, College Park (1970); Colorado State University, Fort Collins, and Imperial College of Science, Technology, and Medicine, London, U.K. (1974); University of California, Davis (1977); INRIA Rochencourt, France (1978); University of Washington, Seattle (1981); Cambridge University, Cambridge, U.K., and the National University of Singapore (1985); University of California, Berkeley (Russell Springer Chair 1987, 1989, and 1991); University of Alberta, Edmonton, Alta., Canada (1992-1994); University of Regensburg, Regensburg, Germany (1993); Toshiba Chair, Institute of Industrial Science, University of Tokyo, Tokyo, Japan (six months from September 1993); University of Maryland (1994); Imperial College of Science, Technology, and Medicine (1995); Technical University of Munich, Munich, Germany (1995); University of Wuerzberg, Wuerzberg, Germany (1996); Chinese University of Hong Kong, and University of Wuerzberg (1997). He has spent periods in industry as a design engineer and as a consultant and has had research grants from industry and government laboratories, including being a named researcher in the Co-Operative Research Centre for Robust and Adaptive Systems. His current research is in control systems, optimization, and signal processing with application areas to robotic and vision systems, GPS/INS navigation, and communication systems. He is co-author, with B. D. O. Anderson, of three books: Linear Optimal Control (Englewood Cliffs, NJ: Prentice-Hall, 1971) Optimal Filtering (Englewood Cliffs, NJ: Prentice-Hall, 1979), and Optimal Control-Linear Quadratic Methods (Englewood Cliffs, NJ: Prentice-Hall, 1989). He is co-author of the following books: Optimization and Dynamical Systems (New York: Springer-Verlag, 1993) (with U. Helmke); Hidden Markov Model Estimation and Control via Reference Methods (New York: Springer-Verlag, 1995) (with L. Aggoun and R. J. Elliott); and High Performance Control (Boston, MA: Birkhauser, 1997) (with T. T. Tay and I. Mareels).

Prof. Moore is a Fellow of the Australian Academy of Technological Sciences and Engineering and a Fellow of the Australian Academy of Science.

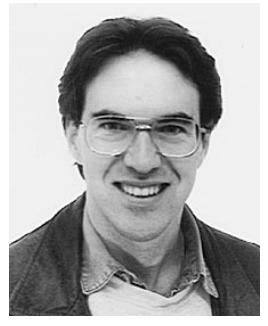

Robert E. Mahony (S'91-M'94) received the B.Sc. degree in mathematics and geology from Australian National University, Canberra, in 1989 before working as a geophysist for two years. Returning to study in 1991, he received the Ph.D. degree in systems engineering from the Research School of Information Sciences and Engineering, Australian National University, in 1994.

Following his study, he worked for the Cooperative Research Center for Robust and Adaptive Systems, a government industrial research organization, for three years. More recently, he has taken a postdoctoral research position at Universté de Technologie de Compiègne, Compiègne, France, which he currently holds. His main research interests are in control of nonlinear motion systems and the study of optimization algorithms in the presence of symmetry. 